\title{
Enhanced recovery ERAS for elderly: a safe and beneficial pathway in colorectal surgery
}

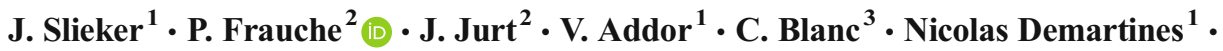 \\ M. Hübner ${ }^{1}$
}

Accepted: 13 October 2016/Published online: 21 October 2016

(C) Springer-Verlag Berlin Heidelberg 2016

\begin{abstract}
Background Enhanced recovery after surgery (ERAS) pathway includes recovery goals requiring active participation of the patients; this may be perceived as "aggressive" care in older patients. The aim of the present study was to assess whether ERAS was feasible and beneficial in older patients. Methods Since June 2011, all consecutive colorectal patients were included in an ERAS pathway and documented in a dedicated prospective database. This retrospective analysis included 513 patients, 311 younger patients ( $<70$ years) and 202 older patients $(\geq 70$ years). Outcomes were adherence to the ERAS pathway, functional recovery, postoperative complications, and hospital stay.

Results Older patients had significantly more diabetes, malignancies, cardiac, and respiratory co-morbidities; both groups underwent similar surgical procedures. Overall adherence to the ERAS pathway was in median $78 \%$ in younger and $74 \%$ in older patients $(P=0.86)$. In older patients, urinary drains were kept longer $(P=0.001)$, and oral fluid intake was reduced from day 0 to day $3(P<0.001)$. There were no differences in mobilization and intake of nutritional supplements.
\end{abstract}

J. Slieker and P. Frauche contributed equally

The content of this manuscript was presented at the Annual Meeting of the Swiss Society of Surgery on the 20th of May 2015, Bern, Switzerland

Nicolas Demartines

demartines@chuv.ch

1 Department of Visceral Surgery, University Hospital CHUV, Lausanne, Switzerland

2 Faculty of Medicine, UNIL University, Lausanne, Switzerland

3 Department of Anesthesiology, University Hospital CHUV, Lausanne, Switzerland
Postoperative complications were similar for both comparative groups ( 51.5 vs. $46.6 \%, P=0.32$ ). Median length of stay was 7 days (IQR 5-13) in older patients vs. 6 days (IQR 4-10) in the younger group $(P=0.001)$.

Conclusion Adherence to the ERAS pathway was equally high in older patients. Despite more co-morbidities, older patients did not experience more complications. Recovery was similar and hospital stay was only 1 day longer than in younger patients. ERAS pathway is of value for all patients and does not need any adaptation for the elderly.

Keywords Enhanced recovery after surgery $\cdot$ Postoperative complications in elderly $\cdot$ Colorectal surgery $\cdot$ Adherence

\section{Introduction}

Enhanced recovery after surgery (ERAS) pathway has been proven to be beneficial for patients and should now be considered as standard of care in colorectal surgery [1, 2]. It allows for lower complication rates and shortened length of hospital stay at reduced costs [3]. Significant improvement has been shown with a high adherence to the pathway [4]. However, a question remains about its applicability to the elderly population: ERAS pathway requires active participation and may be perceived as quite aggressive by older patients. Earlier studies have not specifically assessed safety and suitability of ERAS in older patients compared to younger study populations. More recent studies have started to provide answers to these concerns; they suggest similar gain for older patients regarding rate of complications, recovery time, and length of hospital stay [5-10]. However, actual adherence of older patients to the pathway was not reported, and it was therefore unknown whether adjustments were necessary to suit the specific condition of older patients. 
The aim of the present study was to compare the adherence to ERAS pathway in colorectal patients $<70$ and $\geq 70$ years of age, regarding functional recovery, postoperative complications, and hospital stay.

\section{Methods}

ERAS was systematically introduced for all colorectal surgery at our tertiary academic center in May 2011; all consecutive patients above age 18 were treated within the ERAS pathway, no patient was excluded. The current analysis included all patients operated until February 2014. From April 2012 on, all emergent surgery patients were also routinely included. Therefore, neither a priori nor post hoc selection was performed. Stoma surgery (defined as closure or creation of an end/diverting colo or ileostoma) was included.

The institutional enhanced recovery pathway was published recently [3] and is in accordance with the ERAS recommendations updated in 2013 [11].

Patients were divided in a younger and an older group, with a cut-off for age at 70 years [7]. Prospective documentation of demographic and surgical details of all patients, as well as functional and clinical outcomes until 30 days after surgery was performed on a routine basis for all patients since implementation of the pathway in May 2011. Readmissions were documented from the day of discharge until 30 days postoperatively. Documentation was performed by a trained ERAS nurse.

\section{Compliance with ethical standards}

Informed consent was obtained from all individual participants included in the study. Approval of use of patient information for this study was given by the ethical committee of our institution (CER-VD); under protocol n ${ }^{\circ}$ 10/14.

The study was conducted in accordance with STROBE criteria (http://strobe-statement.org/) and registered under www.researchregistry.com (UIN427).

\section{Outcome measures}

Adherence to the ERAS pathway was assessed by the number of ERAS elements fulfilled out of a total of 15 and was further divided and analyzed as preoperative, perioperative, and postoperative adherence. Non-adherence was defined as adherence to fewer than $70 \%$ of the 15 elements; a univariate analysis was then run to find out which variables influenced non-adherence.

Functional recovery was assessed by return of bowel function, amount of oral intake during first 3 days (deemed sufficient at $1000 \mathrm{ml}$ of fluids per day and $300 \mathrm{kcal}$ of oral nutritional supplements per day) and sufficient mobilization during first 3 days (out of bed on day of surgery and $4 \mathrm{~h}$ or more per day during next days).

Overall complications were assessed during hospital stay; complication rates were defined per patient; a patient could have suffered from one or several complications. A severe complication was regarded as greater than or equal to a grade III Clavien classification [12] and severe complication rates were computed based on the most severe complication for each patient. They were then analyzed non-exhaustively as surgical and non-surgical complications. Post-discharge complications were assessed at 30 days postoperatively and described separately from complications during hospital stay.

At last, length of hospital stay was compared, counting from day of surgery until day of discharge. Readmissions were documented from the day of discharge until 30 days postoperatively. Discharge was allowed based on preestablished criteria (oral pain management, independent mobilization, sufficient food intake, gastrointestinal function, and the absence of a suspicion of complications) [13].

\section{Statistical analysis}

Descriptive statistics for discrete variables were reported as frequency and percentage and continuous variables were reported as median and interquartile range (IQR). Chi-square or Fisher's exact test were used for comparison of discrete variables. Continuous variables were compared with MannWhitney U test. All statistical tests were 2-sided and a level of 0.05 was considered statistically significant. Statistical analyses were performed with SPSS ${ }^{\circledR} 19$ (IBM, Armonk, New York, USA).

\section{Results}

Five hundred thirteen patients were included in the study with 311 patients in the younger group and 202 in the older group.

\section{Demographics and surgical details}

Comparison of baseline characteristics (Table 1) revealed significant differences besides the median age (55 vs. 77 years, $P<0.001)$. Patients in the older group had higher ASA (American Society of Anesthesiologists) scores and WHO (World Health Organization) performance scores and significantly more co-morbidities than their younger counterparts as displayed in Table 1. Surgical details can be found in Table 2. Type of surgery and the surgical approach showed no significant difference between both groups. Emergency surgery was significantly more prevalent in the older group. 
Table 1 Patient characteristics below and above 70 years.

\begin{tabular}{llll}
\hline & $\begin{array}{l}\text { Age }<70 \mathrm{y} \\
n=311\end{array}$ & $\begin{array}{l}\text { Age } \geq 70 \mathrm{y} \\
n=202\end{array}$ & $p$ value \\
\hline Age years (IQR) & $55(46-64)$ & $77(74-83)$ & $<0.001^{*}$ \\
Gender (M/F) & $182 / 129$ & $94 / 108$ & $0.009^{*}$ \\
Body mass index $(\mathrm{kg} / \mathrm{m} 2)$ & $24.8(21.9-28.7)$ & $25.2(22-28.1)$ & 0.984 \\
ASA score & & & $<0.001 *$ \\
$1-2, n(\%)$ & $257(82.9 \%)$ & $120(59.4 \%)$ & \\
$3-4, n(\%)$ & $53(17.1 \%)$ & $82(40.6 \%)$ & 0.054 \\
WHO score & & $176(88 \%)$ & \\
$1-2, n(\%)$ & $288(93.2 \%)$ & $24(12 \%)$ & $33(16.3 \%)$ \\
$3-4, n(\%)$ & $21(6.8 \%)$ & $118(58.4 \%)$ & $0.005^{*}$ \\
Diabetes, $n(\%)$ & $23(7.5 \%)$ & $38(17.9 \%)$ & $<0.001^{*}$ \\
Cardiac history, $n(\%)$ & $73(23.5 \%)$ & $124(61.7 \%)$ & $0.044^{*}$ \\
Respiratory history, $n(\%)$ & $34(10.9 \%)$ & $17(8.4 \%)$ & $<0.001^{*}$ \\
Presence of malignancy, $n(\%)$ & $108(34.8 \%)$ & $<0.001^{*}$ \\
Smoker, $n(\%)$ & $86(27.8 \%)$ & & \\
\hline
\end{tabular}

ASA American Society of Anesthesiologists score, $W H O$ World Health Organization score.

$* p$ value $<0.05$

\section{ERAS protocol adherence}

Overall adherence with the ERAS pathway was in median $78 \%(67-85 \%)$ in younger and $74 \%(64-85 \%)$ in older patients $(P=0.86)$. Adherence to preoperative elements was $100 \%(83-100 \%)$ versus $100 \%(83-100 \%) ; P=0.869$, for intraoperative elements it was $80 \%(80-85 \%)$ vs. $80 \%$ (75$100 \%) ; P=0.822$ and for postoperative elements it was $72 \%$ (76-81\%) vs. $69 \%(52-81 \%) ; P=0.082$. No significant differences were noted for any of the three, pre, per and postoperative phases. Non-adherence ( $<70 \%$ of elements) was found in $20 \%$ of all patients. Old age was not a significant predictor of non-adherence $P=0.308$. However, high ASA score $(P=0.004)$, rectum resections $(P=0.002)$, open surgery $(P=0.016)$, emergency surgery $(P<0.001)$, and duration of surgery $(P=0.002)$ were found to have statistical significance.

Application of individual care elements is displayed in Fig. 1. In the preoperative and intraoperative phase, no significant differences between the groups were observed (Fig. 1a, b). In the postoperative phase (Fig. 1c), urinary catheters were kept longer than the recommended duration (removal at day 1) in older patients $(P=0.001)$ without revealing a difference in the occurrence of urinary retention between the groups. Nasogastric tubes were left in place more often at the end of
Table 2 Surgical details in patients below and above 70 years

\begin{tabular}{llll}
\hline & $\begin{array}{l}\text { Age }<70 \text { y } \\
n=311\end{array}$ & $\begin{array}{l}\text { Age } \geq 70 \text { y } \\
n=202\end{array}$ & $p$ value \\
\hline Main procedure & & & 0.098 \\
Colon resection, $n(\%)$ & $158(50.8 \%)$ & $121(59.9 \%)$ & $44(21.8 \%)$ \\
Rectum resection, $n(\%)$ & $79(25.4 \%)$ & $34(16.8 \%)$ & \\
Stoma procedure, $n(\%)$ & $60(19.3 \%)$ & $3(1.5 \%)$ & 0.561 \\
Other surgery, $n(\%)$ & $14(4.5 \%)$ & $101(50.2 \%)$ & \\
Surgical approach & & $87(43.3 \%)$ & $13(6.5 \%)$ \\
Laparoscopic surgery, $n(\%)$ & $145(46.6 \%)$ & & \\
Open surgery, $n(\%)$ & $139(44.7 \%)$ & $150(74.3 \%)$ & \\
Converted, $n(\%)$ & $27(8.7 \%)$ & $41(20.3 \%)$ & \\
Timing & & $11(5.5 \%)$ & \\
Elective surgery, $n(\%)$ & $263(84.6 \%)$ & $176(120-251)$ & 0.81 \\
Surgery after unplanned admission, $n(\%)$ & $30(9.6 \%)$ & & \\
Acute surgery $(<24 \mathrm{~h}), n(\%)$ & $18(5.7 \%)$ & $193(128-260)$ & \\
Surgery duration $($ minutes), median (IQR) & &
\end{tabular}


Fig. 1 a Detailed elements of the ERAS pathway for postoperative phase. $x$-axis: adherence in percentages. b Detailed elements of the ERAS pathway for peroperative phase. $x$-axis: adherence in percentages $P O N V$ postoperative nausea and vomiting. c Detailed elements of the ERAS pathway for postoperative phase. $x$-axis: adherence in percentages $O N S$ oral nutritional supplements a

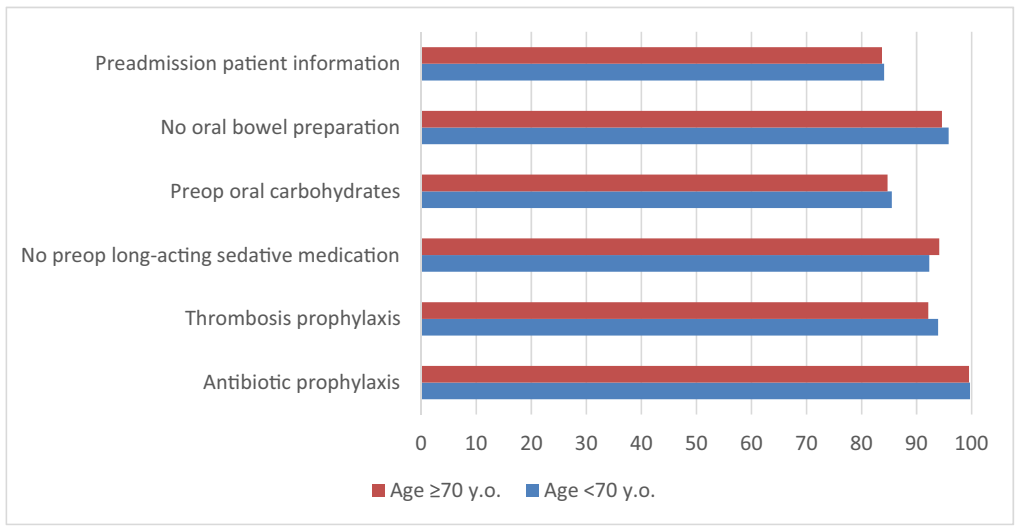

x-axis: adherence in percentages

b

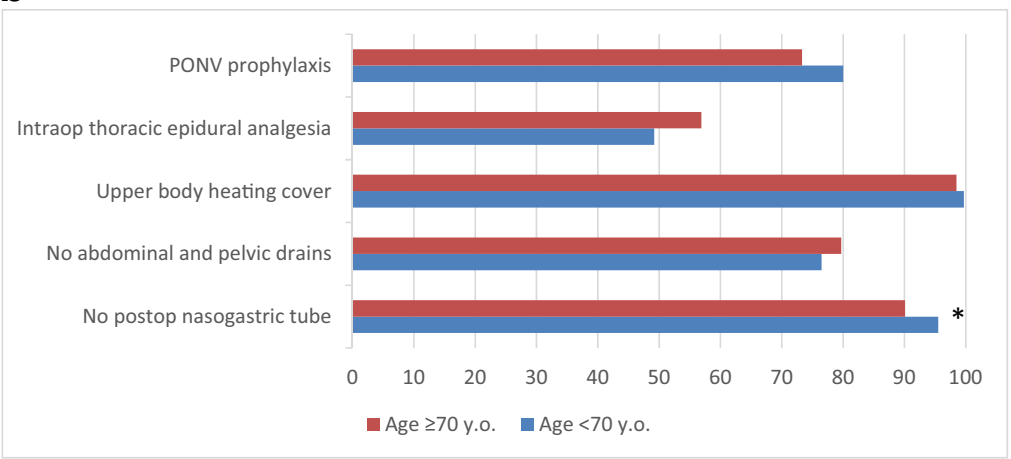

$x$-axis: adherence in percentages

PONV: Postoperative Nausea and Vomiting

*: p-value $<0.05$

C

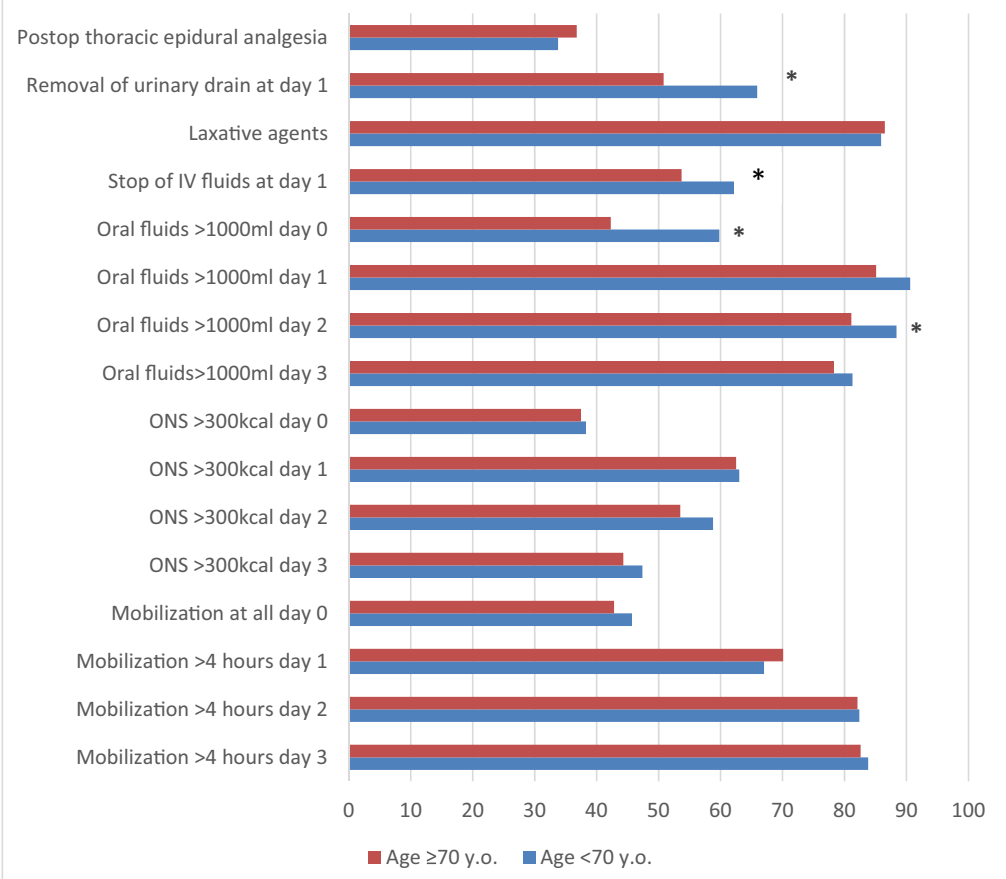

$x$-axis: adherence in percentages

ONS: Oral Nutritional Supplements

*: $p$-value $<0.05$ 
Table 3 Postoperative complications in patients below and above 70 years.

\begin{tabular}{llll}
\hline & $\begin{array}{l}\text { Age }<70 \text { y } \\
n=311\end{array}$ & $\begin{array}{l}\text { Age } \geq 70 \mathrm{y} \\
n=202\end{array}$ & $p$ value \\
\hline Complications during hospitalization, $n(\%)$ & $145(46.6 \%)$ & $104(51.5 \%)$ & 0.320 \\
Severe complications: Clavien III-IV, $n(\%)$ & $47(15.1 \%)$ & $29(14.4 \%)$ & 0.899 \\
Surgical complications, $n(\%)$ & $50(16.1 \%)$ & $37(18.3 \%)$ & 0.548 \\
Anastomotic leaks, $n(\%)$ & $13(4.2 \%)$ & $7(3.5 \%)$ & 0.817 \\
Surgical site infections, $n(\%)$ & $19(6.1 \%)$ & $18(8.9 \%)$ & 0.295 \\
Cardiovascular complications, $n(\%)$ & $12(3.9 \%)$ & $21(10.4 \%)$ & $0.005 *$ \\
Respiratory complications, $n(\%)$ & $24(7.7 \%)$ & $25(12.4 \%)$ & 0.092 \\
Infectious non-surgical complications, $n(\%)$ & $50(16.1 \%)$ & $44(21.8 \%)$ & 0.129 \\
Urinary retentions, $n(\%)$ & $34(11 \%)$ & $27(13.4 \%)$ & 0.485 \\
Complications after discharge, $n(\%)$ & $30(11.8 \%)$ & $19(12 \%)$ & 1.000 \\
Reoperation, $n(\%)$ & $41(13.2 \%)$ & $25(12.4 \%)$ & 0.893 \\
Readmission, $n(\%)$ & $17(5.5 \%)$ & $6(3 \%)$ & 0.199 \\
Mortality, $n(\%)$ & $2(0.6 \%)$ & $6(3 \%)$ & 0.062 \\
\hline
\end{tabular}

$* p$ value $<0.05$

surgery $(P=0.019)$ in older patients, with no significant variance in occurrence of postoperative ileus. Other differences were found in duration of intravenous fluids, and in oral fluid intake although absolute differences were minor $(300 \mathrm{ml})$.

\section{Functional recovery}

Return of bowel function was slower in the older group, with a median of 3 days [2-4] until first stool, versus 2 days [2,3] in the younger group $(P=0.044)$, and 2 days [1-3] versus 2 days $[1,2]$ until first flatus $(P=0.018)$.

\section{Postoperative complications}

Overall, $48.5 \%$ of all patients experienced a complication, $14.8 \%$ of those being severe (Clavien III and IV). No differences were found between the groups for the incidence of overall or severe complications (Table 3). Non-surgical complications, notably cardiovascular problems were more frequent in the older group. Reoperation rate was $12.8 \%$ and readmission rate $4.5 \%$, with no statistical differences between groups. Eight patients died after surgery, six in the older group, and two in the younger group $(P=0.062)$. Causes of death were three cardiac complications, three septic shocks, one respiratory failure, and one renal failure.

\section{Hospital stay}

Hospital stay was significantly longer for older patients: they stayed in median 7 days (IQR 5-13) versus 6 days (IQR 4-10) after surgery $(P=0.001)$.

\section{Discussion}

ERAS pathway for colorectal surgery was applied with the same high adherence for older and younger patients; only minor differences were noted for early mobilization and food intake. Clinical outcomes were comparable for younger and older patients.

Several recent studies have addressed the use of enhanced recovery protocol in the elderly population in colorectal surgery. A systematic review by Bagnall et al. summarizing data from 16 studies including 5965 patients [7] has evaluated and confirmed the safety of ERAS in the elderly population by finding similar prevalence in morbidity and mortality compared to a younger population and slightly longer hospital stays. Despite his own results, Bagnall suggested further studies to measure adherence to the pathway and to evaluate the necessity to tailor the protocol for elderly patients. Comparing younger and older patients, Baek found no difference in postoperative results between patients below and above 70 years following an enhanced recovery protocol [6]. However, adherence to the protocol was not detailed in their study, so it is not known if the same protocol was applied in both groups. Verheijen found a longer length of stay for older patients, but did not examine adherence to the ERAS protocol, either [10]. Only two studies have specifically considered adherence to the protocol. A small study by Kisialeuski comparing enhanced recovery in patients below and above 65 years of age found a trend for less mobilization and longer IV fluids [8]. Feroci found a longer hospital stay, higher morbidity and mortality rate, with a lower adherence to the ERAS protocol in patients older than 75 years [9]. The lower adherence was described for all items: minimal invasive operative techniques, bladder catheter removal, liquid diet tolerance, solid diet tolerance, target mobilization and no usage of drains. These two 
studies are in contradiction with the findings of our present study. We found that despite significantly more co-morbidities, worse disability scores and more emergency procedures in older patients, adherence with the ERAS protocol was equally high; also with regards to early mobilization. Our results strongly suggest that the same pathway should be applied for every patient, with no adjustments in older patients.

Only minor differences were observed in a few postoperative elements. Urinary catheters may have been maintained longer in older patients for fear of urinary retention, or because of a doctor-felt need of urine output measurement to assess renal function. However, we found no differences in urinary retention rates between both groups. Nasogastric tubes were also left in place longer in older patients at the end of surgery, but again there was no difference in occurrence of postoperative ileus. These elements seem to have been subjectively modified by the doctor in charge, but ought to be adapted according to the guidelines as they might impede functional recovery.

Because early oral hydration was slightly impaired in older patients on the first days after surgery, emphasis should be placed on promotion of oral hydration. Assessment of early feeding was only reported by the quantity of oral nutritional supplements taken by patients and not on the quantity of solid food intake, but in this, no differences were found. However, no baseline measurements were available and older patients are known to have a lower fluid intake compared to younger subjects. A surprising point is that older patients, who could be expected to be frailer and harder to mobilize, achieved sufficient mobilization in the early postoperative days and did as well as younger patients.

Return of bowel function was delayed in older patients but the difference was of 1 day only.

Overall complication rate was relatively high at $48.5 \%$ compared to literature. This may be explained by rigorous reporting of all complications including grade I and II, such as urinary retention, postoperative nausea and vomiting, and bedside opening of wounds. The severe complications rate (grade III and IV) of $14.8 \%$ reported in our study is comparable to existing literature. Only non-surgical complications were higher for older patients but this could be expected given the higher rates of co-morbidities in that population. The presumption that following an ERAS pathway and sending patients home earlier would be detrimental for older patients is not confirmed by our results. The rate of readmissions and complications after discharge was no different than with younger patients. The difference in length of hospital stay between both groups was statistically significant, but that being only 1 day, it can be seen as a satisfying result.

One limitation of the study is its retrospective aspect, since the research question was formulated after collection of the data. However, all data were prospectively collected; all patients undergoing surgery were included in the pathway and all patients in the database were analyzed; the risk for selection bias is therefore minimized. Another limitation lies in the choice of a cut-off value for age at 70 years; this choice was made considering that no evidence exists on what the cut-off value for age should be. In existing literature, a third of studies defines this cut-off at 70 years, the rest of studies define it at 75 or 80 years.

In conclusion, this study shows the ERAS pathway in colorectal surgery can be applied without adaptations for older patients. Older patients are worse off initially because of their age and co-morbidities, but are able to follow elements of the pathway that require their active participation almost as well as younger patients. Clinical outcomes were nearly the same for both groups. It can be concluded that ERAS pathway in colorectal surgery is safe and beneficial for the elderly population as well, without limitation.

\section{Compliance with ethical standards}

Conflict of interest The authors declare that they have no conflict of interest.

Ethical approval All procedures performed in studies involving human participants were in accordance with the ethical standards of the institutional and/or national research committee and with the 1964 Helsinki declaration and its later amendments or comparable ethical standards. For this type of study formal consent is not required.

Sources of funding None.

\section{References}

1. Greco M, Capretti G, Beretta L, Gemma M, Pecorelli N, Braga M (2014) Enhanced recovery program in colorectal surgery: a metaanalysis of randomized controlled trials. World J Surg 38(6):15311541

2. Varadhan KK, Neal KR, Dejong $\mathrm{CH}$, Fearon $\mathrm{KC}$, Ljungqvist $\mathrm{O}$, Lobo DN (2010) The enhanced recovery after surgery (ERAS) pathway for patients undergoing major elective open colorectal surgery: a meta-analysis of randomized controlled trials. Clin Nutr 29(4):434 440

3. Roulin D, Donadini A, Gander S, Griesser AC, Blanc C, Hubner M et al (2013) Cost-effectiveness of the implementation of an enhanced recovery protocol for colorectal surgery. The British journal of surgery 100(8):1108-1114

4. Gustafsson UO, Hausel J, Thorell A, Ljungqvist O, Soop M, Nygren J et al (2011) Adherence to the enhanced recovery after surgery protocol and outcomes after colorectal cancer surgery. Arch Surg 146(5):571-577

5. Wang Q, Suo J, Jiang J, Wang C, Zhao YQ, Cao X (2012) Effectiveness of fast-track rehabilitation vs conventional care in laparoscopic colorectal resection for elderly patients: a randomized trial. Color Dis 14(8): 1009-1013

6. Baek SJ, Kim SH, Kim SY, Shin JW, Kwak JM, Kim J (2013) The safety of a "fast-track" program after laparoscopic colorectal surgery is comparable in older patients as in younger patients. Surg Endosc 27(4):1225-1232 
7. Bagnall NM, Malietzis G, Kennedy RH, Athanasiou T, Faiz O, Darzi A (2014) A systematic review of enhanced recovery care after colorectal surgery in elderly patients. Color Dis 16(12):947-956

8. Kisialeuski M, Pedziwiatr M, Matlok M, Major P, Migaczewski M, Kolodziej D et al (2015) Enhanced recovery after colorectal surgery in elderly patients. Wideochir Inne Tech Maloinwazyjne 10(1):30-36

9. Feroci F, Lenzi E, Baraghini M, Garzi A, Vannucchi A, Cantafio S et al (2013) Fast-track surgery in real life: how patient factors influence outcomes and compliance with an enhanced recovery clinical pathway after colorectal surgery. Surg Laparosc Endosc Percutan Tech 23(3):259-265

10. Verheijen PM, Vd Ven AW, Davids PH, Vd Wall BJ, Pronk A (2012) Feasibility of enhanced recovery programme in various patient groups. Int J Color Dis 27(4):507-511
11. Gustafsson UO, Scott MJ, Schwenk W, Demartines N, Roulin D, Francis N et al (2013) Guidelines for perioperative care in elective colonic surgery: Enhanced Recovery After Surgery (ERAS $((\mathrm{R})))$ Society recommendations. World J Surg 37(2):259-284

12. Dindo D, Demartines N, Clavien PA (2004) Classification of surgical complications: a new proposal with evaluation in a cohort of 6336 patients and results of a survey. Ann Surg 240(2):205-213

13. Fiore JF Jr, Bialocerkowski A, Browning L, Faragher IG, Denehy L (2012) Criteria to determine readiness for hospital discharge following colorectal surgery: an international consensus using the Delphi technique. Dis Colon rectum 55(4): $416-423$ 